\title{
La estrategia del análisis teórico de la práctica de profesores de Secundaria en formación: los incidentes críticos
}

\author{
José Fernández González ${ }^{1,3}$, Juan José Marrero Galván ${ }^{2,3}$ \\ ${ }^{1}$ Departamento Didácticas Especificas. Universidad de La Laguna.E-mail: jofdę@ull.es \\ ${ }^{2}$ IES Virgen de la Candelaria. Santa Cruz.de Tenerife. E-mail:jmargalp@gmail.com \\ ${ }^{3}$ Grupo Blas Cabrera Felipe - GITEP
}

[Recibido en noviembre de 2012, aceptado en marzo de 2013]

\begin{abstract}
Se desarrolla una secuencia de trabajo en la preparación del Prácticum del Máster de formación del profesorado de secundaria, consistente en el uso de los incidentes críticos como estrategia de formación de profesores noveles. En una primera fase, los profesores noveles conocen situaciones de la enseñanza en el contexto de análisis de laboratorio, sin riegos en las decisiones y con posibilidad de repetir, lo que les permite aplicar el aprendizaje multidisciplinar del máster. Posteriormente, en una segunda fase, durante la realización del prácticum en centros educativos, se les solicita que recopilen los incidentes críticos que detecten; finalmente, en la fase posterior a las prácticas, analizan algunos de los incidentes que han encontrado.
\end{abstract}

Palabras clave: incidente crítico; metodología; educación secundaria; formación y perfeccionamiento del profesorado.

The strategy of the theoretical analysis of the practice of secondary teachers in training: critical incidents

It develops a sequence of work in the preparation of the Master Practicum teacher training school, consisting in the use of critical incidents as training strategy of novice teachers. In the first phase, new teachers know teaching situations in the context of lab tests, with no risks and be safe in the decisions and replicability, allowing them to apply the learning multidisciplinary master. Subsequently, in a second phase, during the conduct of the practicum in schools, they are required to collect the detected critical incidents and finally, in the post-practice, they discuss some of the incidents found.

Keywords: critical incidents; methodology; secondary education; teacher formation and training.

\section{Introducción}

Se suele considerar que, si se está preparado para hablar sobre un tema, también se puede enseñar. Es decir, puede parecer que para enseñar basta con saber y dominar la asignatura objeto de enseñanza para afrontar el aprendizaje de los alumnos. Desde esta óptica se atiende a los procesos de información como si fueran de enseñanza y se ignoran los procesos de aprendizaje. Por ello, desde hace tiempo se plantea la necesidad de formar profesionales que, además de ser buenos conocedores de su materia, sean capaces de reflexionar sobre lo que enseñan, de tomar decisiones idóneas sobre el planteamiento en el aula y de dar respuestas adecuadas a situaciones educativas nuevas (Fernández, Elortegui y Medina, 2003; Contreras, Monereo y Badía, 2010).

Para conseguir un perfil de profesorado que pueda asumir responsabilidades se hace necesario pensar en una formación continua en una doble vertiente: como aprendiz, seleccionando, elaborando y organizando la información que ha de aprender, y como enseñante, planificando su acción docente, de manera que él ofrezca a sus alumnos, una guía de cómo utilizar los procedimientos de aprendizaje acorde con lo que ha asumido como aprendiz (Monereo, Castelló, Clariana, Palma y Pérez, 1999). 
Si no hay una actuación intencionada del docente, las "estrategias de aprendizaje" no se enseñan o, en todo caso, se practican de forma muy reducida y como una aplicación mecánica y poco reflexiva.

En este sentido, desde la formación inicial y continua, se tendrá que ofrecer a los profesores instrumentos de interpretación y análisis de la situación en la que se desarrolla su actividad, que les permitan tomar decisiones respecto a su actuación como aprendices y como docentes estratégicos, de manera que se vaya enriqueciendo y ampliando su formación en la interacción con la realidad cotidiana de la práctica profesional (Monereo, et. al. 1999; Akkerman y Meijer, 2010). Esto le va a permitir elaborar una identidad profesional docente que le permita responder a ¿qué hacer en un centro de secundaria?. Se suele comparar a los profesores con actores que interpretan un papel (cuyo guión ha escrito otro) y que tiene que motivar, conmover e impactar en sus espectadores para que lo adquirido perdure en el tiempo (Monereo y Monte, 2011). Vilches y Gil (2010) en su trabajo sobre el máster de formación inicial del profesorado de enseñanza secundaria, señalan que "para que el futuro profesor obtenga el máximo provecho de su vivencia del trabajo en el aula y de todo lo que le acompaña (programación de tareas, participación en la gestión del centro, etc.), es preciso que el plan de trabajo y el análisis de lo vivido y realizado responda a una programación diseñada junto al tutor universitario que habrá de implicarse en el seguimiento de estas tareas".

\section{Fundamentación}

\section{Concepto y estructura del incidente crítico}

La problemática que se le suele presentar al profesorado en el aula, cuando se da una situación inesperada, es que se le exige una decisión para las que no suele tener experiencia anterior en la que basarse. Estas situaciones, por ser especialmente difíciles, van a requerir de diferentes soluciones didácticas, esto es: tácticas, técnicas, rutinas, decisiones, posturas corporales, discurso, orientaciones metodológicas, etc. Dichos sucesos los denominamos "incidentes críticos". Suelen ser inesperados, desestabilizadores del discurrir normal y subjetivos a cada docente.

Es importante plantear a los profesores noveles estas propuestas didácticas para que constituyan parte de sus contenidos de formación; es decir, que pasen a formar parte de su aprendizaje significativo.

Coincidimos con Monereo y Monte (2011) cuando indican que los docentes nos adaptamos para responder en distintos contextos, definidos por factores cambiantes, por lo que hemos de saber construir distintas facetas o versiones de la identidad profesional (distintos yoes profesionales) sin abandonar el bagaje disciplinar y didáctico adquirido, que permite dar una respuesta más acomodada a cada contexto; hemos de dilucidar cuál es la mejor versión en cada momento.

Al referirnos a estas situaciones conflictivas se usa el término "incidentes críticos", entendiéndolos como un evento o suceso que, aún cuando es plausible que se produzca en el transcurso de una determinada actividad, por los efectos que ocasiona en alguno de los participantes (sean éstos positivos o negativos), constituyen un acontecimiento o hito destacado en ese contexto (Monereo y Pozo, 2011). En nuestro trabajo para su estudio, los estructuramos de la siguiente forma:

a) Contexto en el que se producen.

b) Descripción de la problemática.

c) Posibles causas que explican lo sucedido. 
d) Soluciones más acordes a los problemas planteados.

e) Reflexión del incidente crítico y las implicaciones de las materias del máster.

El incidente crítico (IC) es una estrategia estructurada y en la cual se presenta a los profesores situaciones escritas de la enseñanza y se les pide que tomen una decisión en función de la información que se les proporciona. Son análisis en situaciones de laboratorio, sin riegos en las decisiones y con posibilidad de repetir, lo que permite a los profesores acercarse a realidad.

En ellos, se tratan algunos aspectos de relatos de situaciones reales, en las que se detalla lo más sobresaliente, para tener un conocimiento cabal del incidente. El análisis de las causas y las posibles soluciones está contextualizado desde la diversidad de planteamientos en los aspectos educativos, que se reflejan de manera diferente según cada uno de los distintos modelos didácticos (Elórtegui, Fernández y Medina, 2004). Es por ello, que las causas y soluciones que se ofrezcan sólo son válidas para la idiosincrasia del grupo que las argumenta. Aunque esto, les resta cierto carácter general como norma, no deja de apreciarse su capacidad formativa para profesores noveles en prácticas, tanto porque les sirve de herramienta de reflexión ante posibles situaciones que se le pueden dar, como elemento interrogante ante casos parecidos.

Coincidimos con Benarroch (2011) cuando señalan como propuesta de mejora, para el máster de formación del profesorado de educación secundaria, "la necesidad de diseñar mecanismos que aseguren que existe coherencia entre los modelos de enseñanza utilizados en la impartición del máster y los que se pretenden que los futuros profesores apliquen en sus aulas". Por tanto, es conveniente reflexionar sobre las implicaciones de los conocimientos adquiridos a través de los campos de formación de las distintas materias del máster en el incidente objeto de estudio.

\section{El incidente crítico como estudio de casos}

Dado que en el estudio de casos se trata de presentar un profesor y/o de un colectivo de profesores para someterlo a estudio por parte de profesores en formación, a través de las opiniones y juicios de éstos y de las discusiones y debates entre ellos, se infieren complejas y significativas interrelaciones de causas y consecuencias que dirigen la conducta humana y las creencias acerca de la enseñanza. Se ha de presentar el caso etnográfico con múltiples y variados datos: visión del mundo del profesor, sus constructos, descripciones de su contexto con estímulo-respuesta de su actuación, observación del caso en clase, notas de campo, fotografías, diarios, programaciones, libros de texto, tareas de los Alumnos, etc. (Fernández y Fernández 1994).

En la didáctica de las Ciencias Experimentales, Mellado y González (2000) indican, que “los estudios de caso combinados con las prácticas de enseñanza, se están utilizando con notable éxito en la formación de profesorado de ciencias", aportando tres procedimientos y que brevemente exponemos:

a) Estudios de caso grabados en vídeo de los propios profesores en formación.

b) Estudios de caso de otros profesores en formación y profesores principiantes.

c) Estudios de caso de profesores de ciencias considerados excelentes.

También señalan: "la escasez de este tipo de estudios, especialmente de profesores considerados excelentes". Sin lugar a dudas se pierde una excelente fuente de conocimiento profesional.

Los incidentes críticos pueden considerarse como un tipo particular de "estudio de casos" tal como se recoge en la bibliografía (Millán, 1997; Moral y Pérez, 1996; Mellado, 1998; Doménech, 1999), ya que son propuestas contextualizadas de situaciones conflictivas reales que, aunque expuestas brevemente y de forma muy estructurada, pretenden que los profesores 
noveles, tras un proceso de análisis, discusión y reflexión, aporten posibles soluciones a las problemáticas planteadas.

\section{El incidente crítico como resolución de situaciones problemáticas}

La búsqueda de la aplicación al mundo real llevó al "positivismo de las Ciencias", es decir, a convertir la capacidad explicativa o predictiva de una teoría en el principal criterio de evaluación de su calidad: la aplicabilidad al mundo real determina la aceptación de cualquier conocimiento por la comunidad.

El reflejo de esta concepción positivista del saber en el conocimiento escolar se percibe en la forma en que se establece la relación entre teoría y práctica en el aula. Esta relación suele reflejarse en dos ámbitos: a) la aplicación de la teoría a supuestos que simulan casos reales y b) la aplicación de la teoría a la interpretación de los datos (o información) que la realidad suministra (Rodrigo, 1994).

Desde una concepción didáctica que integre plenamente los dos ámbitos de aplicación de la teoría (simulación de casos reales e interpretación de datos) se encuentra el trabajo por resolución de situaciones problemáticas (problem solving), en el que se utiliza tanto el análisis y aplicación de conocimientos teóricos en abstracto, como el trabajo sobre diseños experimentales orientados a completar y contrastar el conocimiento disponible, con el fin de solucionar la situación planteada. Dependiendo del modelo didáctico que cada profesor adopte (Lucas, 1993; Fernández, Elórtegui, Rodríguez y Moreno, 1996; Perales, 2000), así serán las actividades que realice con sus alumnos, separando o integrando los dos ámbitos de aplicación de la teoría para la interpretación del mundo real.

La conveniencia de este acercamiento entre lo que sucede en las Prácticas de Enseñanza y la Formación Teórica, ya ha sido puesta de manifiesta con anterioridad por otros autores (Mellado y González, 2000). Igualmente, Marcieux (1987) propone centrar la formación continua del profesorado en las prácticas de clase. En coherencia se propone partir de la "resolución de problemas" profesionales de los profesores.

Así mismo, Imbernón (2007) establece una "orientación de la formación hacia un proceso de provocación de una reflexión basada en la participación (aportación personal, no rigider, motivación, metas comunes, normas claras, coordinación, autoevaluación) y mediante metodología formativa basada en casos, intercambio, debates, lecturas, trabajo en grupo, incidentes críticos, situaciones problemáticas". Por tanto, la formación debe plantearse en íntima conexión con la práctica docente y debe orientarse de modo que favorezca la reflexión en y sobre la práctica (Valcárcel y Sánchez, 2000). Esto no es ajeno al profesorado, si tenemos en cuenta estudios sobre formación de profesorado de Ciencias Experimentales que así lo indican, ya que los docentes muestran preferencia por tratar "temas relacionados con el trabajo real en el aula" frente a otras situaciones como "contenido de la materia", "teoría curricular", "proyectos de reforma", etc. (Marrero, Fernández, Tejera y Elórtegui, 2013).

\section{El incidente crítico desde los modelos didácticos}

Todo docente actúa profesionalmente en función de un conjunto de conocimientos sustentados por unas teorías implícitas que constituyen la base cognitiva de su trabajo. Estas teorías tienen características muy semejantes a las ideas previas de los alumnos descritas abundantemente en la bibliografía: raramente se explicitan, son autoconsistentes, contienen tanto errores como aciertos muy arraigados y tienen un papel fundamental en el aprendizaje de nuevos conocimientos. Los docentes tienen un rol acorde con una teoría compleja y estructurada: su modelo didáctico. La palabra "modelo" aparece como muestra o estereotipo de posible alternativa a la enseñanza aprendizaje, en la línea de cómo se usan los modelos en la 
Ciencia; esto es, de la misma forma que se usan los modelos de Rutherford o de Bohr para el átomo, o el de mosaicos fluidos para la membrana celular. Los modelos didácticos, como cualquiera de los modelos mencionados, interpretan la realidad en un campo de aplicación determinado. Esta interpretación suele ser inexacta fuera de los límites de utilidad, es decir, tienen un rango de validez (Fernández et al., 1996).

Todo docente actúa profesionalmente en función de un conjunto de conocimientos sustentados por unas teorías implícitas que constituyen la base cognitiva de su trabajo. Estas teorías tienen características muy semejantes a las ideas previas de los alumnos descritas abundantemente en la bibliografía: raramente se explicitan, son autoconsistentes, contienen tanto errores como aciertos muy arraigados y tienen un papel fundamental en el aprendizaje de nuevos conocimientos. Los docentes tienen un rol acorde con una teoría compleja y estructurada: su modelo didáctico. La palabra "modelo" aparece como muestra o estereotipo de posible alternativa a la enseñanza aprendizaje, en la línea de cómo se usan los modelos en la Ciencia; esto es, de la misma forma que se usan los modelos de Rutherford o de Bohr para el átomo, o el de mosaicos fluidos para la membrana celular. Los modelos didácticos, como cualquiera de los modelos mencionados, interpretan la realidad en un campo de aplicación determinado. Esta interpretación suele ser inexacta fuera de los límites de utilidad, es decir, tienen un rango de validez (Fernández et al., 1996).

Los incidentes críticos, por sus características, tienen un alto componente subjetivo tanto en su selección como en su análisis: sólo si son relevantes para el observador serán señalados y su relevancia vendrá determinada por la interacción con la visión profesional de quien lo estudia. Por ello, situaciones muy relevantes para unos docentes serán calificadas por otros como carentes de interés, siendo su pensamiento profesional, su modelo didáctico, el marco de análisis que utilizará para clasificarlas de una u otra forma. Se podría partir de los modelos didácticos (Lucas, 1993; Fernández y Elortegui, 1996) para señalar qué considerará cada cual un incidente crítico, cómo lo analizará y qué situaciones considerará irrelevantes.

Finalmente, investigaciones recientes sobre la utilización de incidentes críticos (en adelante IC) en la formación del profesorado (Guzmán, Marín y Zezati, 2011; Bilbao y Monereo, 2011; Nail y Gajardo, 2012), aportan numerosas conclusiones interesantes a tener en cuenta, así por ejemplo Bilbao y Monereo (2011) sugieren:

"Empezar por orientar la formación a esos IC particulares y no a temáticas y principios psicopedagógicos generales, como babitualmente se realiza. Pensamos que la contextualización de esa formación y la inclusión de estrategias de auto-regulación cognitiva y, muy especialmente, emocional, pueden incrementar el bagaje de los docentes para enfrentar con éxito esas situaciones recurrentes y estresantes [...] Dotar a los maestros de herramientas que les permitan aprovechar los IC a los que se enfrentan, para convertirlos en oportunidades de aprendizaje".

Las numerosas investigaciones sobre IC desarrolladas en la en la última década se manifiesta en la cantidad de publicaciones (artículos, libros, comunicaciones en congresos, etc.), aportando en la mayoría de los casos nuevos puntos de vista y optimizando los IC como recurso en la formación inicial y permanente del profesorado. Esto refleja, sin lugar a dudas, su importancia en el campo de la didáctica y justifica su uso en el máster de formación del profesorado de secundaria.

Por tanto, en este trabajo nos marcamos como objetivos:

1. Proponer una secuencia didáctica o de trabajo con profesores en formación, con el fin de que estos puedan desarrollar herramientas que le permitan responder ante determinadas situaciones problemáticas que se le plantean en la acción del docente en el aula. 
2. Mostrar una relación de IC, a modo de ejemplos, que se puedan utilizar en la formación inicial de futuros profesores de enseñanza secundaria.

\section{Metodología de aplicación del incidente crítico}

\section{Justificación}

Durante varios años en las enseñanzas del Curso de Cualificación Pedagógica (CCP) impartido en la Universidad de La Laguna, los profesores noveles que lo cursaban demandaban la falta de relación entre las asignaturas generales y específicas del plan de estudios y la práctica docente en los centros escolares. Esta situación se abordó en el curso 2007-08 a partir de un seminario de formación en el que participaban los docentes implicados, el profesorado de la Universidad y el profesorado de Secundaria (tutores de los profesores noveles), trabajando en varias sesiones, utilizando la técnica del análisis de incidentes críticos (Elórtegui, Medina, Tejera y Fernández, 2006).

Desde entonces, en las Prácticas en los Centros de Secundaria, inicialmente correspondientes al CCP y más tarde en el Máster de Formación de Profesorado de Secundaria de Ciencias Experimentales (en los seminarios organizativos de las prácticas), se ha utilizado esta técnica de trabajo por los profesores noveles, intentando la conexión entre las materias del Máster y su aplicación en la resolución de las situaciones observadas, resultando de gran utilidad en la formación inicial del profesorado. En el proceso de trabajo se demanda del profesorado novel aquellas situaciones que más les han llamado la atención, y sus soluciones apoyadas en las materias que han estudiado en su formación didáctica y pedagógica como profesor, tales como organización escolar, diversidad de alumnado, evaluación, problemas a aprendizaje o de enseñanza, disciplina, convivencia, etc.

En definitiva, se trata de estudiar casos reales que acontecen en el aula, analizar sus causas así como proponer soluciones apoyándose en los conocimientos obtenidos a través de las materias generales y específicas que se imparten en el cuerpo de teórico de su formación.

Es una propuesta de trabajo, siendo una indagación experimental cualitativa basada en un análisis del aprendizaje (en el módulo específico), en la acción del profesorado novel cuando realiza las prácticas en centros de bachillerato.

\section{Secuencia de actividades}

El trabajo con incidentes críticos es apropiado para ejercitar una dinámica de grupo donde el profesorado novel pueda explicitar su posicionamiento acerca de muchos tópicos recurrentes en la docencia.

La secuencia de actividades que se recomienda/propone seguir, con un colectivo de profesores en formación, es la siguiente:

A) Fase previa a las prácticas en centros educativos.

1. Justificación de la importancia de los incidentes críticos y sus implicaciones didácticas.

Se intenta poner en situación acerca de lo que significa la situación en la que suele encontrarse un profesor de aula. Se le presentan situaciones a las que tiene que dar respuesta inmediata, casi sin tiempo para reflexión, que ésta va a estar supeditada a la capacitación, experiencia y formación del docente, que además no siempre se responde igual y que en definitiva la solución final depende del hábitat cultural del profesorado. 
Normalmente los profesores noveles no están familiarizados con la forma de trabajo que le vamos a proponer y su expectativa probablemente sea que el profesorado es quien "le enseña" lo que tiene que hacer en cada situación, sin ser consciente de que la solución no suele servir a todos los docentes por igual. Es necesario emplear parte del tiempo inicial en permitir que los profesores noveles tomen conciencia del método de trabajo y de sus implicaciones didácticas.

2. Presentación de un "incidente crítico" analizado exhaustivamente.

Se presenta el estudio completo de un incidente crítico, realizado por alumnos de cursos anteriores, que orienta a los profesores noveles acerca de cómo aplicar el método de trabajo por primera vez. Se explica su estructura y se profundiza en su contenido.

A modo de ejemplo, se trata el IC de la "dificultad para trabajar en equipo", que desarrollamos en el apartado 4.a de este trabajo y se ofrece un resumen en el cuadro 1.

Cuadro 1. Incidente analizado exhaustivamente.

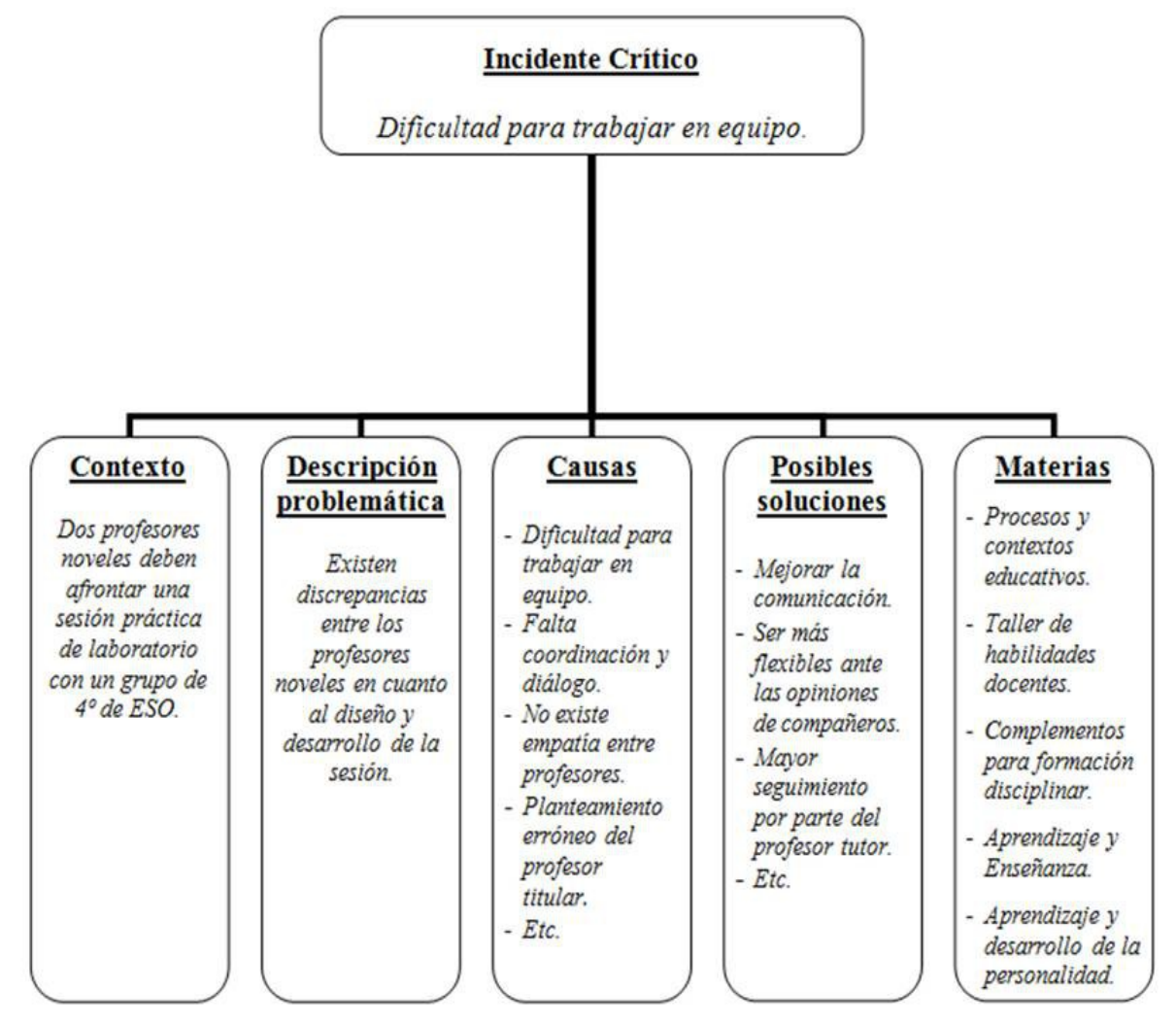

3. Propuesta de un incidente crítico en el que se explica el contexto y la problemática, y se solicita que se debata, en pequeños grupos de trabajo, sobre su posible resolución en el aula, proponiendo causas y soluciones. Finalmente, se realiza una puesta en común y se debate las causas y soluciones adoptadas.

Esta propuesta permite a los profesores noveles ponerse en situación, analizar el incidente en concreto que se le presenta y elaborar sus propias propuestas, así como completar un incidente que no han vivido y prepararse para identificarlos en su experiencia de aula. 
Se propone como ensayo de trabajo la "Divergencia de profesores en el desdoble Biología-Geología", que se comenta en el apartado 4.b.

\section{Preparación de la tarea a desarrollar durante la fase de prácticas.}

Se trata, de explicar la tarea que se va a realizar durante las prácticas en el centro educativo, con el objetivo de consensuar el procedimiento y la ejecución de la misma.

B) Fase de prácticas en los centros educativos.

5. Recopilación de los incidentes críticos que hayan reconocido durante las prácticas en Centro, siguiendo la estructura comentada y ensayada anteriormente, realizan una redacción de los incidentes e insistiendo en detallar el enunciado, tanto el contexto como la descripción de la situación acontecida.

Las semanas de prácticas en centros suelen ser pródigas en incidentes que los profesores noveles deben recopilar. Su condición de profesores noveles, aún muy cerca de los alumnos y la presencia de varios profesores noveles en el aula con el profesorado titular y tutor, les permite ver aspectos e incidentes que normalmente pasan desapercibidos o que se consideran normales. Con frecuencia, su análisis muestra trasfondos mucho más complejos que los mostrados en un primer vistazo superficial.

C) Fase posterior a las prácticas en centros educativos.

6. Selección de algunos de los incidentes recopilados que sean más llamativos para trabajar en pequeños grupos o equipos de trabajo), con el objetivo de que se analicen y se propongan las posibles causas y soluciones (tal como se procedió en el incidente critico analizado en la fase previa).

En esta etapa, siempre posterior a la estancia en los centros docentes, cada equipo de trabajo aporta uno o dos incidentes para su estudio. Cada equipo hace un primer análisis de estos incidentes que sirve de base, posteriormente, al aprendizaje y trabajo en el gran grupo y que puede servir de elemento de evaluación de los profesores noveles.

7. Preparación por parte de los equipos de trabajo de la etapa anterior, de una exposición oral de algunos de estos incidentes para todos sus compañeros, constituyentes del grupo aula.

Esta exposición oral exige a cada equipo una preparación y una reflexión en la que deben utilizar los conocimientos adquiridos y la experiencia que han acumulado, como base para su desarrollo profesional. Simultáneamente, esto permite que afloren concepciones previas profesionales que deben ser explicitadas y revisadas como paso previo a un aprendizaje significativo.

En algunos pequeños grupos, más implicados en la dinámica, se les puede solicitar que analicen qué "materias" del conocimiento pedagógico y didáctico, que se hayan tratado en el cuerpo teórico del Máster de Secundaria están implicadas en el incidente crítico estudiado. Así mismo, podrían aportar, desde la nueva formación adquirida entroncada con su pensamiento profesional, ¿qué haría como profesorado novel?.

8. Realización de una evaluación cualitativa y formativa, por parte del alumnado, de la reflexión del incidente crítico expuesto por cada equipo.

Tanto la exposición del equipo que presenta el incidente, como las aportaciones de los demás equipos presentes, permite el desarrollo de una actividad de evaluación 
individual y colectiva, en la que se señalan las preconcepciones detectadas, los sesgos provocados por los modelos didácticos de cada cual y las alternativas a una misma situación.

Todo este proceso permite a los alumnos la acumulación de experiencias y de posibles pautas de actuación que pasan a ser un acervo profesional utilizable en situaciones reales de aula. No sustituyen a la experiencia de aula, pero permiten que el alumno disponga de recursos profesionales obtenidos tras reflexión que eviten, en lo posible, las reacciones irreflexivas ante la presión de la inmediatez del aula.

Cuadro 2. Resumen de la secuencia de trabajo.

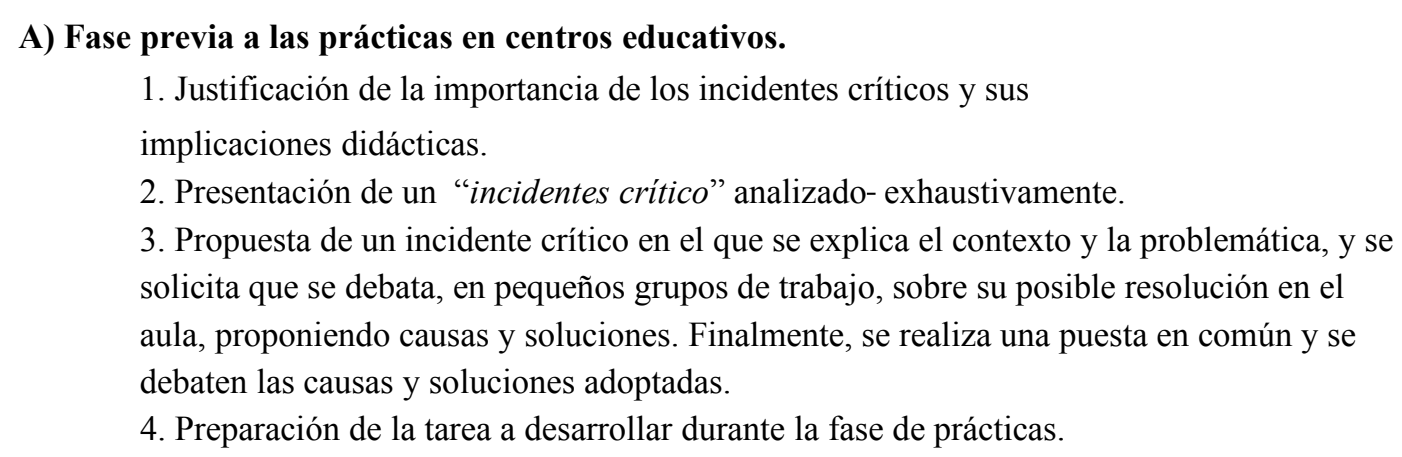

B) Fase de prácticas en los centros educativos.

5. Recopilación de los incidentes críticos que hayan reconocido durante las prácticas en Centro, insistiendo en detallar el enunciado, tanto el contexto como la descripción de la situación acontecida.

C) Fase posterior a las prácticas en centros educativos.

6. Selección de algunos de los incidentes más llamativos recopilados, uno o dos para trabajar en pequeños grupos o equipos de trabajo $\neq$, con el objetivo de que se analicen y se propongan las posibles causas y soluciones.

7. Preparación por parte de los equipos de trabajo de la etapa anterior, de una exposición oral de algunos de estos incidentes para todos sus compañeros, constituyentes del grupo aula.

8. Realización de una evaluación cualitativa y formativa, por parte del alumnado, de la reflexión del incidente crítico expuesto por cada equipo.

\section{Incidentes críticos trabajados}

\section{Incidente crítico analizado exhaustivamente: "Dificultad para trabajar en equipo" \\ Contexto}

Dos profesores noveles, en prácticas en el departamento de "Física y Química de un centro de secundaria, deben realizar una práctica de laboratorio con dos cursos de $4^{\circ}$ de la ESO. Un profesor en prácticas es físico y el otro es químico.

\section{Descripción/problemática}

El primer día de prácticas, en el que los profesores noveles se dedican a la observación, el profesor titular propone realizar una experiencia de laboratorio con los alumnos de $4^{\circ}$ de la ESO sobre el principio de Arquímedes para ser llevada a cabo en la última semana de las prácticas. El profesor titular comenta una serie de posibles prácticas de laboratorio que se pueden llevar a cabo pero concede libertad para elegir la práctica en cuestión. Los profesores 
noveles comentan distintas posibilidades para llevar a cabo la práctica elegida, pero el profesor novel de física ya ha establecido sus esquemas de preparación.

Ante la imposibilidad de realizar conjuntamente el guión de prácticas, cada profesor novel elige un experimento para luego fusionarlos en un mismo guión. Cuando el profesor novel de química muestra su trabajo al compañero, éste ya ha diseñado toda la práctica y la guía del alumno. A la hora de intentar fundir los dos trabajos en una sola práctica, el profesor novel de física se dedica a hacer juicios valorativos sobre la práctica del profesor novel de química y a mutilar el trabajo para incorporar sólo aquello que él considera que está bien. Una vez que se termina el trabajo, el profesor novel de química le sugiere al profesor novel de física que dicho trabajo es excesivo para los alumnos, pero éste hace oídos sordos.

Se lleva a cabo la experiencia con los alumnos, en la que el profesor novel de física acapara la mayor parte del tiempo. Una vez terminada la práctica, y ante las dudas que les surgen a los alumnos, el profesor novel de física decide hacer por su cuenta un informe explicativo para los alumnos, mientras el profesor novel de química no participa por encontrarse ausente. Cuando el profesor novel de química llega se encuentra a su compañero repartiendo el mencionado informe a los alumnos, en el patio, en la hora del recreo.

En la reunión de departamento el profesor novel de química le recrimina la acción y ambos discuten.

\section{Causas}

- El profesor titular no debería haber pedido ese trabajo desde el primer día.

- El profesor titular debería haber esperado unos días hasta comprobar la posibilidad de que los dos profesores noveles trabajaran en equipo.

- El profesor novel de física considera que el experimento es del temario de física y no tiene nada que ver con la química.

- A ambos profesores noveles les cuesta trabajar en equipo.

- Falta de comunicación entre ambos profesores noveles.

- No hay empatía.

\section{Posibles soluciones}

- El profesor titular debe esperar tres o cuatro días para comprobar cómo es la convivencia entre los dos profesores noveles.

- El profesor titular debe estar supervisando continuamente el trabajo que los profesores noveles están realizando y tratar de acercar las posiciones de ambos.

- Los profesores noveles deben ser más flexibles a la hora de llevar a cabo el trabajo e intentar ponerse siempre en el lugar del otro.

- Debe haber siempre una comunicación positiva entre los profesores noveles, haya o no empatía.

\section{Materias implicadas}

- Procesos y contextos educativos.

- Taller de habilidades docentes.

- Complementos para la formación disciplinar.

- Aprendizaje y Enseñanza.

- Aprendizaje y desarrollo de la personalidad. 


\section{Propuesta de Incidente Crítico: "Divergencia profesores desdoble Biología-Geología"}

Contexto

Clase de Biología y Geología de $1^{\circ}$ de Bachillerato. La mitad de la clase se encuentra en el laboratorio con el profesor titular, realizando una práctica de rocas. La otra mitad sigue en el aula realizando un corte geológico.

\section{Descripción}

Surgen explicaciones diferentes sobre un corte geológico entre el profesor titular y el profesor que realiza el desdoble. Se producen discusiones entre ellos en la reunión de Departamento.

Los alumnos de la clase de desdoble acaban confundidos, pues la explicación del tipo de fallas por el profesor del desdoble no coincide con la que da el profesor titular, lo que hace que tengan dificultades a la hora de analizar ese corte geológico.

Un alumno opina que como el que pone la nota es el titular esa debe ser la respuesta que el dirá.

\section{Otros incidentes críticos trabajados}

Se dispone de aproximadamente de medio centenar de incidentes críticos que han sido analizados por el alumnado del máster. Presentamos sólo las propuestas de algunos ejemplos, dadas las limitaciones de extensión de este artículo.

- Actuación de profesora sustituta en una clase de $4^{\circ}$ de ESO.

Contexto: Grupo de alumnos y alumnas de $4^{\circ}$ de la ESO. El profesor titular está de baja y se incorpora una profesora sustituta.

Descripción: La profesora sustituta entra en el aula sin presentarse, pone retrasos a los alumnos que entran tarde y explica a un nivel muy superior al de la clase. En un momento dado delega en la profesora novel, en prácticas del Máster, que controle la clase. Finalmente les da una charla sobre los privilegios que tienen los alumnos y el poco aprovechamiento que hacen de ello.

- Metodología en una clase de Ciencias de la Naturaleza.

Contexto: Curso de $4^{\circ}$ de la ESO de diversificación curricular del ámbito científico tecnológico, con 10 alumnos. Imparte clase una profesora en prácticas del Máster.

Descripción: Los alumnos llegan tarde. La profesora propone una serie de actividades sobre agentes geológicos externos, en concreto sobre los barrancos. Como consecuencia de llegar tarde los alumnos la profesora reparte todas las actividades programadas al mismo tiempo. Organiza en grupos la clase y reparte una copia de las actividades por grupo.

- Transcurso de la "comisión de coordinación pedagógica" (Secundaria).

Contexto: Durante la fase de observación de las prácticas se celebra una reunión de la Comisión de Coordinación Pedagógica (CCP) del centro. La asistencia a reuniones y/o realización de entrevistas con órganos de coordinación docente está establecido como trabajo de observación durante el prácticum. La reunión se celebra antes de la entrega de actas de la $1^{a}$ evaluación con notas, y el tema central de la misma es el fracaso escolar, el absentismo, la matriculación y los problemas de disciplina. El lema declarado del equipo directivo es que "el centro luzca por fuera y por dentro", y la Dirección demuestra en su actitud preocupación, quizás excesiva, por la imagen del centro. Asisten a la reunión, el Director, el Jefe de Estudios, los Jefes de 
Departamento, el Orientador, y todos los profesores en prácticas. Al llegar éstos a la reunión, el Director comenta que les van a acompañar los profesores que están haciendo el prácticum.

Descripción: En la parte final de la reunión, transcurridos unos 45 minutos, el Jefe de Estudios le comenta algo al Director, quien interrumpe a la Secretaria preguntando que si “ ¿hay alguien que está grabando la CCP?”; a continuación comenta que "primero tenían que haber pedido permiso o por lo menos haberlo dicho" y que "hay cosas que, por ejemplo, yo no quiero, o no me interesan que estén grabadas" (una profesora asiente "ni a mi") y "grabar así sin decir nada, no me parece correcto". Se le explica al Director en ese instante que el motivo por el que se graba es "para facilitar la toma de notas, en lugar de hacerlo por escrito".

- Alumno que no entiende.

Contexto: Profesor de Matemáticas que imparte clase a 17 alumnos de $1^{\circ}$ de la ESO, habitualmente muy indisciplinados.

Descripción: Durante la clase se presentan varios incidentes que la interrumpen, (alumnos hablando, no prestan atención, no obedecen al profesor para hacer los ejercicios, etc.) al tratar de realizar un ejercicio un alumno dice que no ha entendido nada y que no sabe cómo hacerlo. El profesor había dado un repaso y había explicado tres veces como realizar el ejercicio.

- La metodología de $4^{\circ}$ de la ESO no sirve en $2^{\circ}$ Bach.

Contexto: El profesor, Licenciado en Químicas, que da clase a los grupos de Física y Química en $4^{\circ}$ de la ESO, también lo hace en $2^{\circ}$ de Bachillerato. Todos ellos son grupos con similar $\mathrm{n}^{\circ}$ de alumnos y composición en relación a sexos.

Descripción: El profesor no obtiene los mismos resultados en ambos niveles, aplicando la misma metodología. En $4^{\circ}$ de la ESO consigue un mayor grado de participación y motivación que en $2^{\circ}$ de Bachillerato.

- Alumnos no traen libros.

Contexto: Clase de $2^{\circ}$ de la ESO de Ciencias de la Naturaleza. Hay alumnos de diferentes edades que sólo esperan cumplir los 16 años para marcharse del Centro.

Descripción: La profesora empieza un tema nuevo y la mayoría de los alumnos no han traído el libro. Cuando la profesora mandaba a leer decían que no tenían el texto.

- Una de las preguntas del examen sólo la entendía el profesor (rechazaban su metodología por innovadora).

Contexto: Examen de Química de $2^{\circ}$ de Bachillerato: problema de aplicación de las reglas estequiométricas.

Descripción: Durante la corrección del examen en clase, la mayoría de los alumnos mostraron su disconformidad en el planteamiento de uno de los problemas. No sabían afrontarlo aun cuando mayoritariamente podrían resolverlo. El profesor consideraba que debían saberlo plantear en base a conocimientos que supuestamente habían aprendido el curso anterior (él no les había dado clase). El profesor optó por no considerar la pregunta y reforzar el déficit de conocimientos en ese aspecto. 


\section{Consideraciones finales}

\section{Aportaciones de los incidentes críticos en la formación inicial docente:}

- Se puede considerar el uso de incidentes críticos como instrumento didáctico para la formación del profesorado.

- Los incidentes críticos pueden ser utilizados como vía de reflexión acerca de lo que acontece en clase.

- El tratamiento de incidentes críticos favorece la integración / relación de las "prácticas de enseñanza en los centros" con el cuerpo de materias teóricas que constituyen el Máster de Formación de Profesorado de Secundaria.

- Su puesta en práctica permite solapar distintas dinámicas de trabajo, como pueden ser: estudio de casos, resolución de situaciones problemáticas, modelos didácticos, etc.

- Se intenta dotar al profesorado nivel de elementos didácticos de apoyo ante la variedad de problemáticas que se puede encontrar en el aula, al ejercer la profesión docente.

- Induce en la formación del profesor la necesidad de tener una práctica que responda a una teoría.

\section{Implicaciones educativas}

Coincidimos con Bilbao y Monereo (2011) cuando indican que "la identificación, análisis y frmación basada en Incidentes Críticos supone un área de investigación e intervención que puede resultar realmente fructifera al abrir una grieta entre las teoria, concepciones, creencias y sentimientos, básicamente implícitos, que sostienen los docentes y que determinan en buena parte sus decisiones y conductas".

Analizar la tipología de Incidentes Críticos, su categorización y la elaboración de herramientas que permitan estudiar la incidencia de los incidentes críticos en la formación permanente de los profesores de secundaria.

\section{Limitaciones}

No se aportan datos empíricos dado que es un trabajo cualitativo de las clases de Ciencias, si bien es una propuesta del análisis teórico de la práctica de secundaria en formación.

Este trabajo ha sido realizado con profesorado del área de ciencias experimentales. Siendo un trabajo de investigación cualitativa en la acción, fijando especial atención al aprendizaje en las distintas materias y su implicación en la acción de aula.

\section{Referencias bibliográficas}

Akkerman, S y Meijer, P. (2011). A dialogical approach to conceptualizing teacher identity. Teaching and Teacher Education, 27(2), 308-319.

Benarroch A. (2011). Diseño y desarrollo del máster en profesorado de Educación secundaria durante su primer año de Implantación. Revista Eureka sobre Enseñanża y Divulgación de las Ciencias, 8 (1), 20-40. En línea en: http://hdl.handle.net/10498/10203.

Bilbao, G. y Monereo, C. (2011). Identificación de incidentes críticos en maestros en ejercicio: propuestas para la formación permanente. Revista Electrónica de Investigación Educativa, 13(1). Consultado 05/11/2012 [En línea]. 
Contreras, C., Monereo, C. y Badía, A. (2010). Explorando en la identidad: ¿Cómo enfrentan los docentes universitarios los incidentes críticos que ocurren en las aulas de formación de futuros profesores?. Estudios pedagógicos, 36(2), 63-81.

Doménech, F. (1999). El Proceso de Enseñanza/Aprendizaje Universitario: Aspectos Teóricos y Prácticos. Castelló de la Plana: Publicaciones de la Universitat Jaume I.

Elórtegui, N., Fernández, J. y Medina, M. (2004). Los incidentes críticos afrontados desde la diversidad del profesorado. XXI Encuentros de Didáctica de las Ciencias Experimentales, Universidad del País Vasco, San Sebastián.

Elórtegui, N., Medina, M., Tejera, C. y Fernández, J. (2006). Formación didáctica inicial de profesores de Secundaria: descripción de una experiencia como convergencia de la cultura docente universitaria y de secundaria. Investigación en la Escuela, 58, 93-101.

Fernández, J. y Elórtegui, N. (1996). Qué piensan los profesores acerca de cómo se debe enseñar. Enseñanza de las Ciencias, 14 (3), 331-342.

Fernández, J. y Elórtegui, N. y Medina, M. (2003). Los incidentes críticos como Estrategia de Formación de Profesores de Ciencias. La Laguna: Servicio de Publicaciones de la Universidad de La Laguna.

Fernández, J., Elórtegui, N., Rodríguez, J.F. y Moreno, T. (1996). De las actividades a las situaciones problemáticas en los distintos modelos didácticos. En R. Jiménez y A.M. Wamba (Eds.), Actas del XVII Encuentro de Didáctica de las Ciencias Experimentales. Huelva: Servicio de Publicaciones de la Universidad de Huelva

Fernández, J. y Fernández, T. (1994). Técnica de trabajo con profesores sobre su práctica docente: Terapia de Knoll. Investigación en la Escuela, 22, 91-104.

Fernández, J., Medina, M. y Elórtegui, N. (2003). Enseñar a profesores de Secundaria con situaciones problemáticas. Revista electrónica de Enseñanza de las Ciencias, 2 (3) [En línea].

Guzmán, I., Marín, R. y Zezati, G. (2011). Los incidentes críticos en la formación / evaluación de competencias docentes: elementos para una práctica reflexiva. XI Congreso Nacional de Investigación Educativa: Procesos de Formación. México [En línea].Consultado 05/11/2012.

Imbernón, F. (2007). 10 ideas clave. La formación permanente del profesorado: nuevas ideas para formar en la innovación y el cambio. Barcelona: Grao.

Lucas, A. (1993). Condicionantes del currículo y aportaciones de la investigación a la práctica de la educación en Ciencias. En C. Palacios, D. Ansoleaga y A. Ajo (Eds.). Diez años de investigación e innovación en Enseñanza de las Ciencias. Madrid: CIDE.

Marcieux, P. (1987). La formación continua de los enseñantes centrada en los problemas prácticos de la clase. Universidad de Verano sobre la Formación Permanente del profesorado en Europa: Experiencias y perspectivas. Madrid: Comisión de las Comunidades Europeas/MEC.

Marrero, J.J.; Fernández, J.; Tejera, C. y Elórtegui, N. (2013). ¿Qué piensa el profesorado de una comunidad autónoma acerca de su formación tras un cambio educativo?. Revista Eureka sobre Enseñanza y Divulgación de las Ciencias, 10(1), 66-84. En línea en: http://hdl.handle.net/10498/14997.

Mellado, V. (1998). El estudio de aula en la formación continuada del profesorado de Ciencias. Alambique, 15, 39-46. 
Mellado, V. y González, T. (2000). La formación Inicial del Profesorado de Ciencias. En F. J. Perales, y P. Cañal, (Eds.). Didáctica de las Ciencias Experimentales. Alcoy: Marfil.

Millán, M. D. (1997). El estudio de caso como estrategia docente. En S. De la Torre, (Eds.), Estrategias de simulación. Barcelona: Octaedro.

Monereo, C., Castelló, M., Clariana, M., Palma, M. y Pérez, M.L. (1999). Estrategias de enseñanz̧a y aprendizaje. Barcelona: Graó.

Monereo, C. y Monte, M. (2011). Docentes en tránsito: Incidentes críticos en Secundaria. Biblioteca de aula. Barcelona: Graó.

Monereo, C. y Pozo, J. (2011). La identidad en Psicología de la Educación: Necesidad, utilidad y límites. Madrid: Narcea.

Moral, C. y Pérez, M. P. (1996). La discusión de estudios de caso de profesores expertos y principiantes como estrategia para favorecer prácticas más reflexivas en la formación del profesor. Bordón, 48 (1), 73-87.

Nail, O. y Gajardo, J. (2012). La técnica de análisis de incidentes críticos: una herramienta para la reflexión sobre prácticas docentes en convivencia escolar. Psicoperspectivas. Individuo y Sociedad, 11 (2), 56-76.

Perales, F.J. (2000). La resolución de problemas. En F.J. Perales y P. Cañal (Coords.), Didáctica de las Ciencias Experimentales. Alcoy: Marfil.

Rodrigo, M. J. (1994). El hombre de la calle, el científico y el alumno: ¿un solo constructivismo o tres?. Investigación en la Escuela, 27, 7-16.

Valcárcel, M. y Sánchez, G. (2000). La formación del profesorado en ejercicio. En F. J. Perales, y P. Cañal, (Coords.). Didáctica de las Ciencias Experimentales. Alcoy: Marfil.

Vilches, A. y Gil, D. (2010). Máster de formación inicial del profesorado de enseñanza secundaria: algunos análisis y propuestas. Revista Eureka sobre Enseñanza y Divulgación de las Ciencias, 7(3), 661-666. En línea en: http://hdl.handle.net/10498/9819. 